\title{
Análise epidemiológica da candidemia e espécies fúngicas envolvidas
}

Epidemiological analysis of candidemia and fungal species involved

Análisis epidemiológico de la candidemia y especies fúngicas involucradas

Lindoaldo Xavier de SOUSA ${ }^{\mathbf{1}}$

Luiza Carla Oliveira SOUSA ${ }^{\mathbf{1}}$

José Henrique de Araújo $\mathbf{C R U Z}^{\mathbf{1}}$

Rauhan Gomes de QUEIROZ ${ }^{1}$

Eduardo Dias RIBEIRO ${ }^{2}$

Julliana Cariry Palhano FREIRE ${ }^{3}$

${ }^{1}$ Graduação em Odontologia, Universidade Estadual da Paraíba (UEPB) 58704-400 Patos - PB, Brasil

${ }^{2}$ Centro de Ciências da Saúde - CCS, Disciplina de Cirurgia Bucomaxilofacial I, Universidade Federal da Paraíba (UFPB) 58051-900 João Pessoa-PB, Brasil Centro de Ciências, Tecnologia e Saúde - CCTS, Disciplina de Prótese Dentária, Universidade Estadual da Paraíba 58429-500 Campina Grande - PB, Brasil

\section{Resumo}

Introdução: A candidemia é uma das infecções nasocomiais mais frequentes a nível mundial e apresenta diferentes espécies de Candida envolvidas com o desenvolvimento dessa patologia. Objetivo: Este estudo analisou os aspectos epidemiológicos e as diferentes espécies de Candida associadas à candidemia. Material e Métodos: Uma revisão de literatura foi realizada, através da leitura de artigos científicos publicados nas bases de dados Pubmed e Scielo nos últimos cinco anos. Utilizou-se as seguintes combinações de descritores: Candidemia, Candidemia e Prevalência, Candidemia e Incidência, Candidemia e Candida. Resultados: A candidemia é a infecção fúngica mais hostil atualmente encontrada e prevalente nas populações estudadas. A população mais afetada são os recém-nascidos. Apresenta altas taxas de mortalidade e disseminação em várias partes do mundo. A nutrição parenteral, a administração de antibióticos de amplo espectro, hospitalização prolongada, cirurgia prévia e colonização por Candida sp são os principais fatores de risco relatados na literatura. Múltiplas espécies fúngicas estão associadas a essa condição, porém C. albicans é a mais predominante, seguida por C. parapsilosis e C. tropicalis. Conclusões: A Candidemia é uma patologia prevalente e apresenta alta incidência e morbimortalidade nas populações estudadas das diferentes partes do mundo. C. albicans é a espécie mais associada, seguida por C. parapsilosis e C. tropicalis.

Descritores: Candidemia; Prevalência; Incidência; Candida.

\section{Abstract}

Introduction: Candidemia is one of the most frequent nasocomial infections worldwide and presents different Candida species involved with the development of this pathology. Objective: This study analyzed the epidemiological aspects and the different Candida species associated with candidemia. Materials and Methods: A review of the literature was carried out by reading scientific papers published in the Pubmed and Scielo databases in the last five years. The following combinations of descriptors were used: Candidemia, Candidemia and Prevalence, Candidemia and Incidence, Candidemia and Candida. Results: Candidaemia is the most hostile fungal infection currently found and prevalent in the populations studied. The most affected population is newborns. It has high rates of mortality and spread in various parts of the world. Parenteral nutrition, administration of broad spectrum antibiotics, prolonged hospitalization, previous surgery and colonization by Candida sp are the main risk factors reported in the literature. Multiple fungal species are associated with this condition, but C. albicans is the most predominant, followed by C. parapsilosis and C. tropicalis. Conclusions: Candidemia is a prevalent pathology and presents high incidence and morbidity and mortality in the populations studied from different parts of the world. C. albicans is the most associated species, followed by C. parapsilosis and C. tropicalis.

Descriptors: Candidemia; Prevalence; Incidence; Candida.

\section{Resumen}

Introducción: La candidemia es una de las infecciones nasocomiales más frecuentes a nivel mundial y presenta diferentes especies de Candida involucradas en el desarrollo de esta patología. Objetivo: Este estudio analizó los aspectos epidemiológicos y las diferentes especies de Candida asociadas a la candidemia. Material y Métodos: Una revisión de literatura fue realizada, a través de la lectura de artículos científicos publicados en las bases de datos Pubmed y Scielo en los últimos cinco años. Se utilizaron las siguientes combinaciones de descriptores: Candidemia, Candidemia y Prevalencia, Candidemia e Incidencia, Candidemia y Candida. Resultados: La candidemia es la infección fúngica más hostil actualmente encontrada y prevalente en las poblaciones estudiadas. La población más afectada son los recién nacidos. Presenta altas tasas de mortalidad y diseminación en varias partes del mundo. La nutrición parenteral, la administración de antibióticos de amplio espectro, hospitalización prolongada, cirugía previa y colonización por Candida sp son los principales factores de riesgo reportados en la literatura. Múltiples especies fúngicas están asociadas a esta condición, pero C. albicans es la más predominante, seguida por C. parapsilosis y C. tropicalis. Conclusiones: La Candidemia es una patología prevalente y presenta alta incidencia y morbimortalidad en las poblaciones estudiadas de las diferentes partes del mundo. C. albicans es la especie más asociada, seguida por C. parapsilosis y C. tropicalis.

Descriptores: Candidemia; Prevalencia; Incidencia; Candida.

\section{INTRODUÇÃO}

\section{A candidemia é uma das infecções} nasocomiais mais frequentes a nível mundial e está associada a alta morbidade e mortalidade nos países em desenvolvimento, embora a prevalência exata não seja conhecida devido à falta de dados epidemiológicos sistemáticos desses países. Os estudos apontam uma alta incidência de candidemia e um espectro diferente de espécies de Candida $^{1,2}$.

Essa patologia é uma preocupação crescente em todo o mundo, e sua distribuição de espécies tem demonstrado a presença Candida não albicans nas últimas décadas, especialmente em pacientes com malignidade $^{3}$. As cepas formadoras de biofilmes estão associadas a taxas de mortalidade ainda maiores e um mau prognóstico para o paciente ${ }^{1}$. Vários desafios existem no controle dessa doença, entre eles: infraestrutura deficiente; comprometimento das práticas de controle de infecção e dos cuidados de saúde; uso indevido e excessivo de antibióticos e esteroides; indisponibilidade em muitas áreas de testes de diagnóstico avançado e medicamentos antifúngicos; má adesão à terapia antifúngica e ao programa de administração de medicamentos ${ }^{2}$. É necessária uma investigação detalhada sobre os fatores contribuintes e o tratamento adequado ${ }^{4}$.

Considerando a alta morbibmortalidade da candidemia relatada nas pesquisas, esse estudo analisou através de uma revisão de literatura, 
aspectos epidemiológicos como a prevalência, incidência e mortalidade, assim como as diferentes espécies fúngicas envolvidas no desenvolvimento dessa doença.

\section{MATERIAL E MÉTODO}

Realizou-se uma revisão de literatura de artigos científicos publicados nas bases de dados Pubmed e Scielo, nos últimos cinco anos. As seguintes combinações de descritores foram utilizadas: Candidemia, Candidemia AND Prevalence, Candidemia AND Incidence, Candidemia AND Candida. A análise dos títulos e resumos foi criteriosamente realizada por dois pesquisadores no total de 1.046 estudos e 22 artigos foram selecionados para leitura do texto completo e incluídos na presente revisão. Os artigos incluídos apresentaram a investigação da candidemia, com avaliação das variáveis: prevalência, incidência e/ou espécies fúngicas mais encontradas. Não houve distinção de país ou participantes avaliados e foram publicados nos últimos cinco anos. Excluíram-se os estudos que não se enquadraram ao tema proposto e ao período analisado. Os dados coletados foram tabulados no programa Excel for Windows (versão 13.0) de acordo com o ano de publicação.

\section{RESULTADOS E DISCUSSÃO}

Os resultados da pesquisa foram compilados e se apresentam na Tabela 1. Foram selecionados 22 estudos publicados entre os anos 2015 a 2018.

\section{- Candidemia e sua epidemiologia}

A candidose é a infecção fúngica invasiva mais encontrada em todo o mundo. A população mais afetada por candidemia são os neonatos, como afirmam Benedict et al. ${ }^{7}$ e o Sistema Nacional de Vigilância de Infecções Nosocomiais (NNIS), porém essa taxa de incidência vem declinando nos últimos anos devido a métodos como a profilaxia antifúngica. A variação geográfica é evidente entre os casos de candidemia em diferentes partes do mundo, reconhecendo a importância dos dados epidemiológicos para facilitar o tratamento. Caggiano et al. ${ }^{10}$ observaram que Candida albicans foi a espécie dominante na Europa e América; espécies não albicans predominam na Ásia (75\%).

Dentre os recém-nascidos com peso ao nascer $<1000 \mathrm{~g}, 4-8 \%$ desenvolvem candidemia, com uma mortalidade de $30 \% 7$. A mortalidade bruta para pacientes com câncer com candidemia foi relatada em até $30 \%$, cerca de duas vezes maior do que em pacientes com infecções bacterianas na corrente sanguínea ${ }^{1}$.

\section{- Fatores de risco prevalentes}

Diversos fatores de riscos independentes foram encontrados nos estudos, neles estão presentes as características clínicas equivalentes associadas aos pacientes cirúrgicos como: pacientes oncológicos com e sem metástase, em casos dos tumores sólidos como doença de base, cirurgias recentes, nutrição parenteral e uso de cateter ${ }^{16,17,22,24}$.

Hospitalização prolongada e colonização por Candida spp estão envolvidos no desenvolvimento da candidemia ${ }^{25}$. Pacientes com espécies não albicans demonstram ser mais propensos a ter nutrição parenteral do que aqueles com C. albicans ${ }^{10}$.

Outras razões apontadas nos estudos de Fu et al. ${ }^{11}$ e Siri et al..$^{21}$ são a ventilação mecânica e a CVC (remoção de cateter venoso central) a longo prazo, sinalizados como elementos que aumentam a proliferação fúngica. Quando se fala em idosos, o risco de candidemia associado a outras morbidades é ainda maior. Condições como insuficiência renal, diabetes mellitus, doenças cardiovasculares e pulmonares crônicas são os fatores de risco mais presentes nos idosos candidêmicos ${ }^{5,26}$.

- Espécies fúngicas envolvidas

Cepas formadoras de biofilme estão associadas a taxas de mortalidade ainda maiores e prognóstico ruim para o paciente ${ }^{1}$. C. albicans é o principal patógeno da candidemia em todo o mundo, mas a mudança em favor de espécies de Candida não albicans está ocorrendo nos últimos anos. C. albicans foi a espécie fúngica mais prevalente nas diversas regiões do mundo, seguida por C. parapsilosis ou C. tropicallis ${ }^{4,7,9,12,16,19,23}$. O estudo de Caggiano et al. ${ }^{10}$ realizado na Itália mostrou que a espécie mais encontrada nos episódios de candidemia foi C.parapsilosis seguida de C.albicans, e a duração da hospitalização na Unidade de Terapia Intensiva Neonatal de pacientes com C.não albicans foi significativamente maior do que naqueles com C.albicans.

A morte de pacientes por candidemia causada pela $C$. glabrata foi observada em uma maior proporção em relação às outras espécies de Candida, conforme observado no estudo de Barchiesi et al. ${ }^{5}$, realizado em um hospital universitário na Itália.

Outro tipo de Candida spp que está em emergência em âmbito hospitalar é C. auris. Esse patógeno foi recentemente descoberto, é uma espécie altamente resistente e associado a alta mortalidade como foi descrito no estudo de Navalkele et al. ${ }^{27}$. Spivak e Hanson ${ }^{28}$ relataram a alta resistência desse patógeno associado a uma considerável mortalidade dos pacientes.

\section{- Tratamento e Prevenção da Candidemia}

O diagnóstico precoce é a melhor forma de prevenção. A coleta de hemocultura é um procedimento básico em qualquer paciente com suspeita de infecção sistêmica por Candida $s p^{29}$.

A importância do tratamento antifúngico prévio e apropriado, e a aplicação da dose adequada em um curto espaço de tempo se mostrou efetivo ${ }^{30}$. Foi observado que o fluconazol e o voriconazol podem ser aplicados contra a grande parte das espécies de Candida, incluindo $C$. albicans, 
C. parapsilosis e C. tropicalis. Entretanto, a atividade reduzida do fluconazol requer uma atenção para algumas espécies como C. glabrata e a C. krusei $^{31}$.

Tabela 1. Estudos selecionados e classificados quanto autor/ano, tipo de pesquisa, país/local da pesquisa, prevalência, incidência, mortalidade e espécies fúngicas mais prevalentes

\begin{tabular}{|c|c|c|c|c|c|c|}
\hline $\begin{array}{c}\text { Autor/ } \\
\text { Ano }\end{array}$ & $\begin{array}{l}\text { Tipo de } \\
\text { Pesquisa }\end{array}$ & $\begin{array}{c}\text { País/ } \\
\text { Local } \\
\text { da Pesquisa }\end{array}$ & PR & INC & MRT & $\begin{array}{l}\text { Espécies } \\
\text { fúngicas mais } \\
\text { prevalentes }\end{array}$ \\
\hline $\begin{array}{l}\text { Treviño- } \\
\text { Rangel } \\
\text { et al.1 (2017) }\end{array}$ & $\begin{array}{l}\text { Observacional } \\
\text { Retrospectivo }\end{array}$ & $\begin{array}{c}\text { México } \\
\text { Hospital } \\
\text { Universitário }\end{array}$ & 218 & - & $36 \%$ & $\begin{array}{l}\text { C. topicalis }(52,8 \%) \\
\text { eC. albicans } \\
\text { ( } 30,4 \%)\end{array}$ \\
\hline $\begin{array}{l}\text { Wual.3. } \\
(2017)\end{array}$ & $\begin{array}{l}\text { Observacional } \\
\text { Retrospectivo }\end{array}$ & $\begin{array}{c}\text { Taiwan } \\
5 \text { Centros } \\
\text { Médios de } \\
\text { Taiwan }\end{array}$ & 346 & - & - & $\begin{array}{l}\text { C.tropicallis } \\
(41,9 \%)\end{array}$ \\
\hline $\begin{array}{l}\text { Vasiyeva et } \\
\text { al.4(2018) }\end{array}$ & $\begin{array}{l}\text { Prospectivo } \\
\text { Multicêntrico }\end{array}$ & Rússia & 284 & - & - & $\begin{array}{l}\text { C. albicans }(43,2 \%), \\
\text { Cparassilosis } \\
(20,2 \% 6)\end{array}$ \\
\hline $\begin{array}{l}\text { Barchiesiet } \\
\text { al.5(2016) }\end{array}$ & $\begin{array}{l}\text { Observacional } \\
\text { retrospectivo }\end{array}$ & $\begin{array}{l}\text { Itália Hosppitval } \\
\text { Universitiario } \\
\text { da Itália }\end{array}$ & 270 & $27 \%$ & - & $\begin{array}{l}\text { C. parapsilosis } \\
\text { complex (23\%), } \\
\text { C. albicins (54) } \\
\text { C.tropicalis }(4 \%)\end{array}$ \\
\hline $\begin{array}{l}\text { Barchiesi et } \\
\text { al. }{ }^{6}(2017)\end{array}$ & $\begin{array}{l}\text { Observacional } \\
\text { Retrospectivo. }\end{array}$ & $\begin{array}{l}\text { Itália Hospital } \\
\text { Universitário de } \\
\text { Ancona }\end{array}$ & 302 & - & $39 \%$ & $\begin{array}{l}\text { C. allicans } \\
(53 \%) \\
\text { C. prarapsilosis }(23 \% \\
\text { eC.tropicalis } \\
(11 \%)\end{array}$ \\
\hline $\begin{array}{l}\text { Benedict et } \\
\text { al.7(2018) }\end{array}$ & Observacional & $\begin{array}{c}\text { EUA - } 4 \text { áreas } \\
\text { metropolitanas } \\
\text { dos EUA }\end{array}$ & 307 & - & $13 \%$ & $\begin{array}{l}\text { C. albicanss (68\%) } \\
\text { C.prapasilosis } \\
\text { (37\%) } \\
\text { C.abicans }(48.5 \% \text { ) }\end{array}$ \\
\hline $\begin{array}{l}\text { Bhattacharjee } \\
\text { et at.8 (2016) }\end{array}$ & $\begin{array}{l}\text { Observacional } \\
\text { Retrospectivo }\end{array}$ & $\begin{array}{l}\text { India Hospital } \\
\text { Terciário Kolkata }\end{array}$ & 70 & $\begin{array}{c}6,9 \text { por } \\
1000\end{array}$ & . & 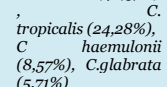 \\
\hline $\begin{array}{l}\text { Breda et al.9 } \\
\text { (2017) }\end{array}$ & $\begin{array}{l}\text { Observacional } \\
\text { Retrospectivo }\end{array}$ & $\begin{array}{l}\text { Brasil } \\
1 \text { hospital publico } \\
1 \text { hospital privado }\end{array}$ & 149 & - & - & $\begin{array}{l}\text { C., alicicans }(52 \%), \\
\text { C. tropicalis }(15 \%) \\
\text { C. glabrata (15\%) }\end{array}$ \\
\hline $\begin{array}{l}\text { Caggiano et } \\
\text { al.1. }(2017)\end{array}$ & $\begin{array}{l}\text { Observacional } \\
\text { Retrospectivo }\end{array}$ & $\begin{array}{c}\text { Ittalia } \\
\text { Hospital } \\
\text { Universitário }\end{array}$ & 41 & $\begin{array}{c}3 \text { para } \\
100\end{array}$ & - & $\begin{array}{l}\text { C. parapsilosis } \\
\left(588,5^{\circ}\right) \text { e C.albicans } \\
(34,1 \%)\end{array}$ \\
\hline $\begin{array}{l}\text { Fue tal.11 } \\
(2017)\end{array}$ & $\begin{array}{l}\text { Observacional } \\
\text { Retrospectivo }\end{array}$ & $\begin{array}{ll}\text { China } & \\
\text { NIIU } & \text { (The } \\
\text { Nonatal } & \text { Thtensive } \\
\text { Unite) } & \text { Care }\end{array}$ & 69 & $8.7 \%$ & - & $\begin{array}{l}\text { C.glabrata (33.3\%), } \\
\text { C.tropicalis } \\
\text { (20.3\%), } \\
\text { C.parasilosis } \\
\text { (1.4\%) } \\
\text { C. kefyr (1.4\%) }\end{array}$ \\
\hline $\begin{array}{l}\text { Guzzetti et } \\
\text { al. } .^{12}(2017)\end{array}$ & $\begin{array}{c}\text { Retrospectivo } \\
\text { Descritivo }\end{array}$ & $\begin{array}{l}\text { Argentina } \\
\text { Hospital Infantil } \\
\text { "Superiora Sor } \\
\text { Maria Ludovica" }\end{array}$ & 117 & - & - & $\begin{array}{l}\text { C. albicans ( }(45 \%) \\
\text { c. parapsilosis } \\
(28 \%)\end{array}$ \\
\hline $\begin{array}{l}\text { Kofteridis } \\
\text { et al.13 (2017) }\end{array}$ & $\begin{array}{l}\text { Caso-controle } \\
\text { Retrospectivo }\end{array}$ & $\begin{array}{l}\text { Grécia } \\
\text { Hospital } \\
\text { Universitário de } \\
\text { Heraklion }\end{array}$ & 106 & - & - & $\begin{array}{l}\text { Não albicans: } \\
\text { C. } \\
\text { (33\%), parapsilosis } \\
\text { C. glabrata (29\%) } \\
\text { C. tropicalis (26\%) }\end{array}$ \\
\hline $\begin{array}{l}\text { Kubiak et } \\
\text { al. }{ }^{14(2015)}\end{array}$ & Observacional & EUA & & 302 & $27,5 \%$ & $\begin{array}{l}\text { C. allicicans }\left(45^{\circ}\right) \text {, } \\
\text { C. parapsilosis } \\
\left(2,2,5^{\circ}\right) \text { e C glabrata } \\
\left(11,3^{\circ}\right)\end{array}$ \\
\hline $\begin{array}{l}\text { Li et al. } 15 \\
(2013)\end{array}$ & $\begin{array}{l}\text { Observacional } \\
\text { Retrospectivo }\end{array}$ & $\begin{array}{l}\text { China } \\
\text { Tianjin Medical } \\
\text { University } \\
\text { Cancer } \\
\text { Institute } \\
\text { and Hospital }\end{array}$ & 80 & $30 \%$ & - & 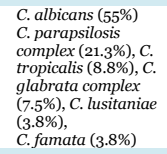 \\
\hline $\begin{array}{l}\text { Lie et al.16 } \\
(2017)\end{array}$ & $\begin{array}{l}\text { Caso-controle } \\
\text { Retrospectivo }\end{array}$ & $\begin{array}{l}\text { China } \\
\text { Instituto e } \\
\text { Hospital do } \\
\text { Câncer da } \\
\text { Universidade } \\
\text { Médica de } \\
\text { Tianjin }\end{array}$ & 80 & - & $30 \%$ & $\begin{array}{l}\text { C. albicans (55\%)e } \\
\text { C.parassilosis } \\
\text { (21,3\%) }\end{array}$ \\
\hline $\begin{array}{l}\text { Lortholary et } \\
\text { al.17 (2017) }\end{array}$ & $\begin{array}{l}\text { Observacional } \\
\text { Retrospectivo }\end{array}$ & $\begin{array}{c}\text { Paris } \\
\text { Hospital-based } \\
\text { surveillance } \\
\text { program }\end{array}$ & 3417 & $58.3 \%$ & - & $\begin{array}{l}\text { C. glabrata (77.\%), } \\
\text { C.parapsilosis } \\
\text { (7.), } \\
\text { C.tropicalis } \\
\text { (8.4\%), } \\
\text { C. allicans (o.8\%) }\end{array}$ \\
\hline $\begin{array}{l}\text { Lovero et } \\
\text { al.1. }(2016)\end{array}$ & Retrospectivo & $\begin{array}{l}\text { Itália Hospital } \\
\text { Universitiario } \\
\text { na Itália }\end{array}$ & 57 & $30 \%$ & - & $\begin{array}{l}\text { C. albicans }(47 \%), \text {, } \\
\text { parapsilosis }(44 \%)\end{array}$ \\
\hline $\begin{array}{l}\text { Márquez } \\
\text { et al.19 } \\
(2017)\end{array}$ & $\begin{array}{l}\text { Observacional } \\
\text { Retrospectivo }\end{array}$ & $\begin{array}{l}\text { Chile } \\
\text { Hospital de base } \\
\text { Valdivia }\end{array}$ & 27 & $\begin{array}{c}0,3 \mathrm{a} \\
0,7 / 1.00 \\
0\end{array}$ & - & $\begin{array}{l}\text { C. albicans } \\
(59,3 \%) e \\
\text { C.troicalis } \\
\text { (14,8\%) }\end{array}$ \\
\hline $\begin{array}{l}\text { Pinhati et } \\
\text { al. }{ }^{20}(2016)\end{array}$ & Transversal & $\begin{array}{l}\text { Brasil } \\
\text { Hospital Santa } \\
\text { Luzia }\end{array}$ & 40 & $45 \%$ & - & $\begin{array}{l}\text { C. parapsilosis } \\
\text { (7o\%) } \\
\text { C. albicans } \\
\text { (22.5\%) } \\
\text { C. glabrata (5\%) }\end{array}$ \\
\hline $\begin{array}{l}\text { Siri } \\
\text { et at. } \\
(2017)\end{array}$ & $\begin{array}{l}\text { Observacional } \\
\text { Retrospectivo }\end{array}$ & $\begin{array}{l}\text { Chile } \\
\text { Hospital } \\
\text { Unviversitário em } \\
\text { Santiago }\end{array}$ & 120 & - & $31,7 \%$ & 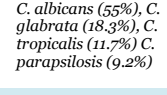 \\
\hline $\begin{array}{c}\text { Spierset } \\
\text { al.22 (2018) }\end{array}$ & $\begin{array}{l}\text { Observacional } \\
\text { Retrospectivo }\end{array}$ & Irlanda do Norte & ${ }_{131}$ & 1.09 & - & - \\
\hline $\begin{array}{l}\text { Tiraboschi et } \\
\text { al. }{ }^{23}(2017)\end{array}$ & $\begin{array}{l}\text { Observacional } \\
\text { Retrospectivo }\end{array}$ & $\begin{array}{l}\text { Argentina } \\
\text { Hospital } \\
\text { Universitário } \\
\text { em } \quad \text { Buenos } \\
\text { Aires }\end{array}$ & 374 & $\begin{array}{l}2,21 / \\
1.000\end{array}$ & - & $\begin{array}{l}\text { C. albicans }(40,9 \%) \text {, } \\
\text { Cparaspsilosis } \\
(21,7 \%)\end{array}$ \\
\hline
\end{tabular}

$\mathrm{PR}=$ Prevalência; $\mathrm{INC}=$ Incidência; MRT $=$ Mortalidade

Fluconazol e anfotericina B são as drogas antifúngicas de escolha usadas na profilaxia e tratamento de Candida spp em recém-nascidos ${ }^{32}$. Anfotericina B, azóis e as equinocandinas são os medicamentos para o tratamento de candidemias. Devido ao aparecimento de cepas intrinsecamente resistentes $(C . \quad$ krusei $) \mathrm{e}$ à sensibilidade diminuída $(C$. glabrata $)$ ao fluconazol $\mathrm{e}$ às equinocandinas ( $C$. parapsilosis), é indispensável conhecer a espécie e monitorar a resistência aos antifúngicos de isolados de Candida $s p^{33}$.

O fluconazol é uma forma alternativa no tratamento de pacientes com candidemia, devido a sua efetividade. Contudo, Pinhati et al. ${ }^{20}$ em seu estudo, demonstra que mais da metade dos pacientes tratados com o fluconazol sofreram resistência ao seu emprego. Devido a essa barreira, Kubiak et al. ${ }^{14}$, em sua observação, fez a mudança do fluconazol para equinocandina ou polieno no tratamento dos pacientes com sensibilidade.

\section{CONCLUSÃO}

Candidemia apresenta alta incidência e morbimortalidade nas diferentes partes do mundo. Estudos observacionais que envolvem sua epidemiologia são os mais realizados. Nutrição parenteral, administração de antibióticos de amplo espectro, hospitalização prolongada, cirurgia anteriores e colonização por Candida $s p$ são os principais fatores de risco relatados na literatura. $C$. albicans demonstrou ser a espécie mais prevalente seguida de $C$. parapsilosis.

\section{REFERÊNCIAS}

1. Treviño-Rangel RJ, Peña-López CD, HernándezRodríguez PA, Beltrán-Santiago D, González GM. Association between Candida biofilmforming bloodstream isolates and the clinical evolution in patients with candidemia: An observational nine-year single center study in Mexico. Rev Iberoam Micol. 2018;35(1):11-16.

2. Kaur H, Chakrabarti A. Strategies to Reduce Mortality in Adult and Neonatal Candidemia in Developing Countries. J Fungi (Basel). 2017;3(3):41.

3. Wu PF, Liu WL, Hsieh MH, Hii IM, Lee YL, Lin YT et al. Epidemiology and antifungal susceptibility of candidemia isolates of nonalbicans Candida species from cancer patients. Emerg Microbes Infect. 2017;6(10):e87.

4. Vasilyeva NV, Raush ER, Rudneva MV, Bogomolova TS, Taraskina AE, Fang Y et al. Etiology of invasive candidosis agents in Russia: a multicenter epidemiological survey. Front Med. 2018;12(1):84-91.

5. Barchiesi F, Orsetti E, Osimani P, Catassi C, Santelli F, Manso E. Factors related to outcome of bloodstream infections due to Candida parapsilosis complex. BMC Infect Dis. 2016;16:387.

6. Barchiesi F, Orsetti E, Mazzanti S, Trave F, Salvi A, Nitti C, Manso E. Candidemia in the elderly: What does it change? PLoS One. 2017;12(5):e0176576. 
7. Benedict K, Roy M, Kabbani S, Anderson EJ, Farley MM, Harb S et al. Neonatal and pediatric candidemia: results from population-based active laboratory surveillance in four US locations, 2009-2015. J Pediatric Infect Dis Soc. 2018;7(3):e78-e85.

8. Bhattacharjee P. Epidemiology and antifungal susceptibility of Candida species in a tertiary care hospital, Kolkata, India. Curr Med Mycol. 2016;2(2):20-7.

9. Breda GL, Tuon FF, Meis JF, Herkert PF, Hagen F, de Oliveira LZ, Dias VC, da Cunha CA, Queiroz-Telles F. Breakthrough candidemia after the introduction of broad spectrum antifungal agents: A 5-year retrospective study. Med Mycol. 2018;56(4):406-15

10. Caggiano G, Lovero G, De Giglio O, Barbuti G, Montagna $\mathrm{O}$, Laforgia $\mathrm{N}$ et al. Candidemia in the Neonatal Intensive Care Unit: a retrospective, observational survey and analysis of literature Data. Biomed Res Int. 2017;2017:7901763.

11. Fu J, Ding Y, Wei B, Wang L, Xu S, Qin P, Wei L, Jiang L. Epidemiology of Candida albicans and non-C.albicans of neonatal candidemia at a tertiary care hospital in western China. BMC Infect Dis. 2017;17(1):329.

12. Guzzetti LB, Vescina CM, Gil MF, Gatti BM. Candidemias en pediatría: distribución de especies y sensibilidad a los antifúngicos [Candidemia in Pediatrics: Species distribution and antifungal susceptibility]. Rev Argent Microbiol. 2017;49(4):320-22.

13. Kofteridis DP, Valachis A, Dimopoulou D, Andrianaki AM, Christidou A, Maraki S, Spernovasilis NA, Samonis G. Factors Influencing Non-albicans Candidemia: A CaseCase-Control Study. Mycopathologia. 2017; 182(7-8):665-72.

14. Kubiak DW, Farmakiotis D, Arons V, Hollins RM, Rostas SE, Weiser LM et al. Utility of inhouse fluconazole disk diffusion susceptibility testing in the treatment of candidemia. Diagn Microbiol Infect Dis. 2016;84(3):223-26.

15. Li D, Zhang W, Zheng S, Ma Z, Zhang P, Liu Z. Surveillance study of candidemia in cancer patients in North China. Med Mycol. 2013;51(4):378-84.

16. Li D, Xia R, Zhang Q, Bai C, Li Z, Zhang P. Evaluation of candidemia in epidemiology and risk factors among cancer patients in a cancer center of China: an 8-year case-control study. BMC Infect Dis. 2017;17(1):536.

17. Lortholary O, Renaudat C, Sitbon K, DesnosOllivier M, Bretagne S, Dromer F; French Mycoses Study Group. The risk and clinical outcome of candidemia depending on underlying malignancy. Intensive Care Med. 2017; 43(5):652-62.
18. Lovero G, De Giglio O, Montagna O, Diella G, Divenuto F, Lopuzzo M, Rutigliano S, Laforgia N, Caggiano G, Montagna MT. Epidemiology of candidemia in neonatal intensive care units: a persistent public health problem. Ann Ig. 2016;28(4):282-87.

19. Márquez F, Iturrieta I, Calvo M, Urrutia M, Godoy-Martínez P. Epidemiología y susceptibilidad antifúngica de especies causantes de candidemia en la ciudad de Valdivia, Chile [Epidemiology and antifungal susceptibility of species producing candidemia in Valdivia, Chile]. Rev Chilena Infectol. 2017;34(5):441-46.

20. Pinhati HM, Casulari LA, Souza AC, Siqueira RA, Damasceno CM, Colombo AL. Outbreak of candidemia caused by fluconazole resistant Candida parapsilosis strains in an intensive care unit. BMC Infect Dis. 2016;16(1):433.

21. Siri L, Legarraga P, García P, González T, Rabagliati R. Cambios clínicos y epidemiológicos de candidemias en pacientes adultos desde 2000 a 2013. Rev Chilena Infectol. 2017;34(1):19-26.

22. Spiers R, Smyth B, Lamagni T, Rooney P, Dorgan $\mathrm{E}$, Wyatt $\mathrm{T}$ et al. The epidemiology and management of candidemia in Northern Ireland during 2002-2011, including a 12-month enhanced case review. Med Mycol. 2019;57(1):23-9.

23. Tiraboschi IN, Pozzi NC, Farías L, García S, Fernández NB. Epidemiología, especies, resistencia antifúngica y evolución de las candidemias en un hospital universitario de Buenos Aires, Argentina, durante 16 años [Epidemiology, species, antifungal resistance and outcome of candidemia in a university hospital in Buenos Aires, Argentina for 16 years]. Rev Chilena Infectol. 2017;34(5):431-40.

24. Vena A, Bouza E, Valerio M, Padilla B, PañoPardo JR, Fernández-Ruiz $M$ et al. Candidemia in non-ICU surgical wards: comparison with medical wards. PLoS One. 2017;12(10):e0185339.

25. Kelly MS, Benjamin DK Jr, Smith PB. The epidemiology and diagnosis of invasive candidiasis among premature infants. Clin Perinatol. 2015;42(1):105-17, viii-ix.

26. Wu JQ, Zhu LP, Ou XT, Xu B, Hu XP, Wang X et al. Epidemiology and risk factors for nonCandida albicans candidemia in non-neutropenic patients at a Chinese teaching hospital. Med Mycol. 2011;49(5):552-55.

27. Navalkele BD, Revankar S, Chandrasekar P. Candida auris: a worrisome, globally emerging pathogen. Expert Rev Anti Infect Ther. 2017;15(9):819-27.

28. Spivak ES, Hanson KE. Candida auris: an Emerging Fungal Pathogen. J Clin Microbiol. 2018;56(2):e01588-17. 
29. Colombo AL, Guimarães T, Sukienik T, Pasqualotto AC, Andreotti R, Queiroz-Telles F et al. Prognostic factors and historical trends in the epidemiology of candidemia in critically ill patients: an analysis of five multicenter studies sequentially conducted over a 9-year period Intensive Care Med. 2014;40(10):1489-98.

30. Gehring GM, Carrilho CMM, Pelisson M, Perugini M, Tano ZN. Candidemia: Revisão Bibliográfica. J Infect Control. 2015;4(4):1-19.

31. Lepak A, Andes D. Fungal sepsis: optimizing antifungal therapy in the critical care setting. Crit Care Clin. 2011;27(1):123-47.

32. Pappas PG, Kauffman CA, Andes DR, Clancy CJ, Marr KA, Ostrosky-Zeichner L et al. Clinical Practice Guideline for the Management of Candidiasis: 2016 Update by the Infectious Diseases Society of America. Clin Infect Dis. 2016;62(4):e1-50.

33. Wisplinghoff H, Ebbers J, Geurtz L, Stefanik D, Major $\mathrm{Y}$, Edmond $\mathrm{MB}$ et al. Nosocomial bloodstream infections due to Candida spp. in the USA: species distribution, clinical features and antifungal susceptibilities. Int $\mathbf{J}$ Antimicrob Agents. 2014;43(1):78-81.

\section{CONFLITO DE INTERESSES}

Os autores declaram não haver conflitos de interesse.

\section{AUTOR PARA CORRESPONDÊNCIA}

Profa. MSc. Julliana Cariry Palhano Freire

Avenida Universitária, s/n - Jatobá

58708-110 Patos - PB, Brasil

Tel: (83) 3511-3000

e-mail: jullianapalhano@hotmail.com 